\title{
Ask the Expert
}

\section{Sexual Health Education Disparities in Asian American Adolescents}

\section{Tsui-Sui Kao \\ Column Editor: Betsy M. McDowell}

Ask the Expert provides research-based answers to practice questions submitted by JSPN readers.

Question: I have noticed that there are increasing numbers of Asian American adolescents in the United States. Even though Asian American youth are doing great academically, their level of sexual health-related knowledge is not explicitly known or understood because of the differences in their cultural backgrounds. What do I need to know in order to ensure that my assessment of their sexual health knowledge is culturally responsive?

Tsui-Sui Kao responds: Asian Americans comprise close to $4.2 \%$ of the total U.S. population. Research on the health status of Asian Pacific Americans (APA) often analyzes the population as one homogeneous group; however, the APA community includes more than 30 diverse ethnic subpopulations that vary by national origin, language, culture, citizenship, and economic status. The main subgroups include Chinese, Japanese, Korean, Filipino, Vietnamese, Asian Indian, and other cultures from Southeast Asia (U.S. Department of Commerce [USDOC], 2000). Even though as a whole, Asian Americans seem to have comparable educational backgrounds and median family incomes with Caucasian Americans, Asian Americans tend to have more family members working at the same time to maintain their financial status and lifestyle. It is important to note that $14 \%$ of the APA population lives below the poverty line, compared to $13 \%$ of the U.S. population, and that Asian Americans are less likely to use federal or state-funded health care programs such as Medicaid (National Asian Pacific American Women's Forum [NAPAWF], 2005).

Asian Americans often are perceived as a model minority, appearing to have abundant resources. In reality, their health is compromised by this misconception, and their health problems are often blamed on the conservative nature of their cultures. Healthcare disparities exist not only in new immigrants but also in the subsequent generations of Asian American children.
Most Asian American children are immigrants themselves or are children of immigrants who straddle two cultures. Few studies are available to help nurses see some of the problems that Asian American adolescents encounter as a result of this bicultural identification. Nurse professionals confront increasing challenges to care for this vulnerable population, especially with the cultural sensitivity to sexual health issues (Kibria, 2002).

Nurses must be cognizant of the importance of Asian Americans' family-centered cultural values, more specifically the taboo against sex education, and the possible impacts of bicultural straddling. Grunbaum, Lowery, Kann, and Pateman (2000) noted that Asian American adolescents tend to delay sexual intercourse, but that once sexually active, they were as likely to have used alcohol or drugs or fail to use a condom during intercourse as any other ethnic group. These factors put them at greater risk for compromised sexual health; therefore, it is important for pediatric nurses to assess the sexual health of all Asian American adolescents.

\section{Asian American Adolescents' Sexual Health}

Studies show that Asian American adolescents tend to delay the onset of sexual intercourse compared to other ethnic groups (Grunbaum et al., 2000; Horan \& DiClemente, 1993; Schuster, Bell, \& Kanouse, 1996; Schuster, Bell, Nakajima, \& Kanouse, 1998; Upchurch, Levy-Storms, Sucoff, \& Aneshensel, 1998); however, they are less likely to receive sexual health-related services prior to and even after becoming sexually active (National Asian Women's Health Organization [NAWHO], 1997; Schuster et al., 1996) than other population groups. As a result, Asian American women tend to be diagnosed with more advanced stages of cervical cancer and breast cancer than Caucasian American women (Frisch \& Goodman, 2000; Hedeen, White, \& Taylor, 1999), and cervical cancer is the leading cause of death for Vietnamese American women (Ishida, 2001). Furthermore, new technology and research show that cervical cancer is actually caused by the human papillomavirus (HPV) (Ordonez, Espinosa, Sanchez- 


\section{Ask the Expert}

Gonzalez, Armendariz-Borunda, \& Berumen, 2004), one of the most prevalent sexually transmitted infections (STIs). Therefore, when focusing on the sexual health of Asian American adolescents, nursing professionals should target cervical cancer detection at the same time.

Many obstacles deter Asian American adolescents from receiving proper sexual health care, such as healthcare providers' misconceptions, stereotyping, racism, sexism, and bicultural gaps between the adolescents' home culture and environmental culture. The disparities in receiving sexual healthcare services exist not only in Asian American women, but are also found in Asian American men. In a survey conducted by the National Asian Women's Health Organization, 89\% of the 802 Asian American men surveyed had never received sexual or reproductive healthcare services, even though $87 \%$ reported having had at least one sexual partner in the past year (NAWHO, 1999).

\section{Bicultural Straddling}

Some research suggests that Asian Americans' reluctance to utilize sexual health-related care is rooted in their conservative cultural background (Horan \& DiClemente, 1993; Okazaki, 2002; Schuster et al., 1998). Still, other research has linked health behaviors with the mistrust of healthcare providers (NAWHO, 1999). A survey of 669 Asian Americans found that despite their comparatively higher socioeconomic status (as measured by income and education), Asian Americans reported having a poorer quality of health care than the overall population. Compared with $62 \%$ of the overall population, only $45 \%$ of Asian Americans were very satisfied with their health care (USDOC, 2000).

One of the reasons for the growing problem of sexual health disparities might be associated with Asian American families' reluctance to talk about sexual health-related issues at home. According to a survey of Asian American women, one-third of the women surveyed never discussed pregnancy, STIs, birth control, or sexuality in their households (NAWHO, 1997). More than half of the women were uncomfortable discussing reproductive health with their mothers and even more uncomfortable discussing these concerns with their fathers and brothers. As a result of this cultural taboo, Asian American girls are often at increased risk for engaging in compromising sexual health-related behaviors. For example, Asian American women have the highest increase in certain STIs, such as gonorrhea and HIV/AIDS (Foo, 2002), yet they are the least likely of all minority groups to believe they are at risk and the least likely to receive sexual health care (Foo, 2002; National Council of Negro Women, 1992; USDOC, 2000).

One possible reason that sex education is a taboo subject in Asian American homes may be the fact that in the traditional Asian culture, sex education was minimized in the schools, and parents, as well as health professionals, were reluctant to discuss sexuality and sexual information (Chan, 1986). Hence, it is possible that Asian American parents lack sexual knowledge themselves, and that may discourage them from approaching the subject with their children. Even though there is no documented study to validate Asian American parents' lack of knowledge, nurses must be aware of this possibility and be more attuned to assessing the Asian American parents' and their adolescents' sexual health-related knowledge, as well as their respective comfort talking to each other about sexual health-related issues and/or concerns.

A recent study of Asian American adolescents linked adolescents' sexual activity with gender, acculturation, and parental attachment (Hahm, 2005). Among Asian American girls, the most acculturated group was three times more likely to have reported sexual intercourse than the least acculturated group. For both boys and girls, a high level of parental attachment was associated with lower odds of sexual intercourse. Hence, nurses need to give particular attention to assessing the level of acculturation among Asian American girls and build on the strengths of a healthy parent-child relationship (Hahm). 


\section{Culturally Responsive Interventions}

Even though second-generation Asian Americans might have less trouble adapting to American society in terms of language, they are not necessarily without difficulties. Straddling between two cultures is not an easy task for these teens because they also are struggling with adolescent development. It is important for nurses to understand that living within two cultures in one society can profoundly affect the growth and development of the Asian American adolescent (Noda, 1989).

In order to provide culturally responsive care to Asian American adolescents, nurse professionals must first be sensitive to their cultural differences and be nonjudgmental in order to build trust with both parents and adolescents. Nurses must approach taboo subjects such as sexual health carefully and respectfully. If possible, a same-gender healthcare worker is preferred when discussing sexual health issues with an Asian American teen to minimize embarrassment.

Additionally, nurses need to educate Asian American parents about sexual health before their children reach adolescence. With the conservative nature of Asian culture, parents' lack of knowledge might become an obstacle for adolescents to develop or learn correct information related to sexual health. This in turn may compromise the adolescents' health development. Providing sexual health knowledge for Asian American parents in a culturally sensitive manner would empower them to help their teens make healthier choices.

Nurses also need to understand that Asian Americans' family-centered values may be utilized as a protective factor for the adolescents' sexual health if nurses can facilitate communication between parents and adolescents. Research has indicated that Asian American parental expectations of their adolescents is a factor in preventing alcohol abuse (Hahm, Lahiff, \& Guterman, 2003) and promoting academic achievement (Hall, 2002). One can assume that parental expectations have the potential to be a protective factor against engaging in risky sexual behaviors. Nurses, therefore, should promote bonding and effective parenting skills in
Asian American families, which in turn can inject parental expectations into adolescents' decision making regarding sexual health.

To summarize, the Asian American adolescent's sexual health has been ignored for a long period of time. Even though there are general beliefs that Asian Americans are model minorities, they are not without difficulties. Healthcare disparities exist not only in populations with low socioeconomic status, but also in the populations who are underutilizing healthcare systems for various reasons. Nurses need to realize that healthcare disparities exist in the Asian American population despite the fact that their Asian American patients have presentable appearances, good educational backgrounds, and/or families with fairly good incomes. Once healthcare disparities in sexual health knowledge are acknowledged, pediatric nurses can provide care that is culturally responsive to the needs of Asian American adolescents, a growing segment of the American population.

\section{Tsui-Sui (Annie) Kao, MS, RN Doctoral Student University of Michigan Ann Arbor, MI}

Author contact: anniekao@umich.edu, with a copy to the Editor: roxie.foster@uchsc.edu

If you would like to submit a question for consideration in the Ask the Expert column, please e-mail your question to the column editor at bmcdowell@lander.edu.

Search terms: Adolescence, Asians, healthcare disparities, sexuality

\section{References}

Chan, D.W. (1986). Sex misinformation and misconceptions among Chinese medical students in Hong Kong. Archives of Sexual Behavior, 19, 73-93.

Foo, L.J. (2002). Asian American women: Issues, concerns, and responsive human and civil rights advocacy. New York: The Ford Foundation.

Frisch, M., \& Goodman, M.T. (2000). Human papillomavirusassociated carcinomas in Hawaii and the mainland U.S. Cancer, 88, 1464-1469. 


\section{Ask the Expert}

Grunbaum, J.A., Lowery, R., Kann, L., \& Pateman, B. (2000). Prevalence of health risk behaviors among Asian American/Pacific Islander high school students. Journal of Adolescent Health, 27, 322-330.

Hahm, H.C. (2005, January). Gender and acculturation differences in Asian American adolescents' sexual activity. Paper presented at the Society for Social Work and Research, Celebrating a Decade of SSWR, Miami, FL.

Hahm, H.C., Lahiff, M., \& Guterman, N.B. (2003). Acculturation and parental attachment in Asian American adolescents' alcohol use. Society for Adolescent Medicine, 33, 119-129.

Hall, G.C.N. (2002). Asian American psychology. Washington, DC: American Psychology Association.

Hedeen, A.N., White, E., \& Taylor, V. (1999). Ethnicity and birthplace in relation to tumor size and stage in Asian American women with breast cancer. American Journal of Public Health, 89, $1248-1252$

Horan, P.F., \& DiClemente, R.J. (1993). HIV knowledge, communication, and risk behavior among White, Chinese-, FilipinoAmerican adolescents in a high-prevalence AIDS epicenter: A comparative analysis. Ethnicity \& Disease, 3, 97-105.

Ishida, D. (2001). Making inroads on cancer prevention and control with Asian Americans. Seminars in Oncology Nursing, 17(3), 220-228.

Kibria, N. (2002). Becoming Asian American: Second-generation Chinese and Korean American identities. Baltimore, MD: Johns Hopkins University Press.

National Asian Pacific American Women's Forum. (2005). Medicaid and Asian Pacific American women. Retrieved April, 19, 2005, from http://modelminority.com/article279.html.
National Asian Women's Health Organization. (1997). Expanding options: A reproductive and sexual health survey of Asian American women. San Francisco, CA: Author.

National Asian Women's Health Organization. (1999). The Asian men's health survey: Sharing responsibilities. San Francisco, CA: Author.

National Council of Negro Women. (1992). The 1991-1992 women of color reproductive health poll (Vol. 55). Washington, DC: Communications Consortium Media Center.

Noda, K.E. (1989). Growing up Asian in America. Boston, MA: Beacon.

Okazaki, S. (2002). Influences of culture on Asian Americans' sexuality. Journal of Sex Research, 39(1), 34-41.

Ordonez, R.M., Espinosa, A.M., Sanchez-Gonzalez, D.J., ArmendarizBorunda, J., \& Berumen, J. (2004). Enhanced oncogenicity of Asian American human papillomavirus 16 is associated with impaired E2 repression of E6/E7 oncogene transcription. Journal of Genetics and Virology, 85(Pt 6), 1433-1444.

Schuster, M.A., Bell, R.M., \& Kanouse, D.E. (1996). The sexual practices of adolescent virgins: Genital sexual activities of high school students who have never had vaginal intercourse. American Journal of Public Health, 86, 1570-1576.

Schuster, M.A., Bell, R.M., Nakajima, G.A., \& Kanouse, D.E. (1998). The sexual practices of Asian and Pacific Islander high school students. Journal of Adolescent Health, 23, 221-231.

Upchurch, D.M., Levy-Storms, L., Sucoff, C.A., \& Aneshensel, C.S. (1998). Gender and ethnic differences in the timing of first sexual intercourse. Family Planning Perspectives, 30, 121-127.

U.S. Department of Commerce. (2000). U.S. Bureau of Census data 2000. Washington, DC: Author. 